\title{
THE METHOD OF LIMITS IN PHYSICS.
}

\author{
By G. B. Blati, \\ Oregon Agricultural College.
}

Certain formulas used in physics may be derived by geometrical methods only by the introduction of the method of limits. The writer has observed that, in these derivations, with possible rare exceptions, the authors of textbooks on elementary physics fail to make clear to the student that the methods used are perfectly rigorous mathematically, and that the formulas obtained are exactly and accurately true, and not merely approximate expressions.

A case in point is the derivation of the equation for the acceleration in uniform circular motion. If a body is moving with uniform velocity in a circular path, a constant force must act upon it, the direction of the force being always toward the center of the path. Whenever a force acts so as to change the velocity of a body, an acceleration is produced in the same direction as that of the force which produces it. In this case, the acceleration is equal tc $\frac{v^{2}}{r}$, where $v$ is the velocity of the body in its path and $r$ is the radius of the path. A fair example of the way in which this expression for the central acceleration is ustally derived is given in the following paragraph.

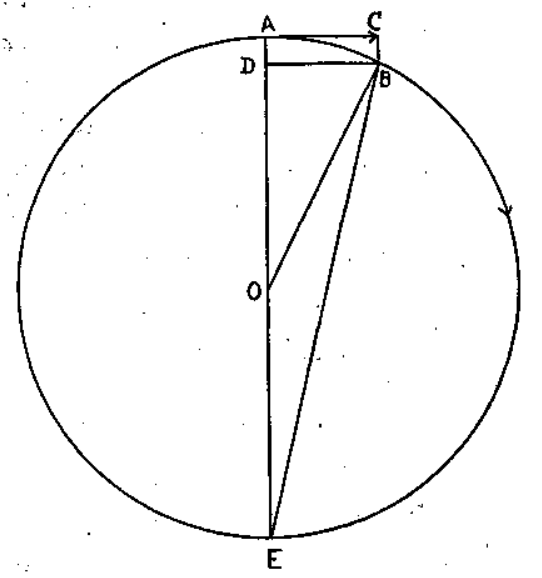

Figure I.

"The fact that the acceleration given by the central force is $\frac{v^{2}}{r}$ can be obtained from a consideration of Figure 1. Suppose a body of mass $M$ to be moving around the circle whose center is $O$, with a uniform velocity, $v$. The space $A B$, over which it passes in the time $t$, is $\mathrm{S}=v t$. Let the time $t$ be taken as a very short time - so short that the $\operatorname{arc} A B$ is practically equal to the chord $\mathrm{AB}$. On $\mathrm{AB}$ as a diagonal, complete the rectangle, ADBC. The distance the body is drawn away from $A C$ toward $O$, by the constant centripetal force, is practically equal to 


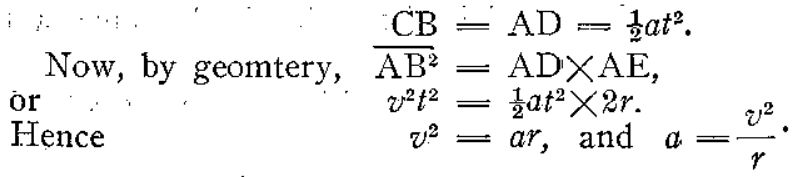

Observe that the author of the proof given above leads the student to believe that the result that he obtains is an approximate one. He says, "Let the time $t$ be taken as a very short time - so short that the arc $\mathrm{AB}$ is practically equal to the chord $\mathrm{AB}$," and again, "The distance which the body is drawn away from $\mathrm{AC}$ is practically equal to CB." It is not wonderful that the student feels dissatisfied with a proof like this. $\mathrm{He}$ can see no essential difference between the argument here and that used to demonstrate that the complete period of the pendulum is expressed by the equation,

$$
\mathrm{T}=2 \pi \sqrt{\frac{l}{g}}
$$

Where $l$ is the length of the pendulum and $g$ is the acceleration of gravity.

As a matter of fact, equation (1) is exactly true, while (2) is only a close approximation to the truth. The following proof, while not rigorously mathematical in the terms used, attempts to lead the student to see that the acceleration at the point $\mathrm{A}$ is accurately, and not approximately, $\frac{v^{2}}{r}$.

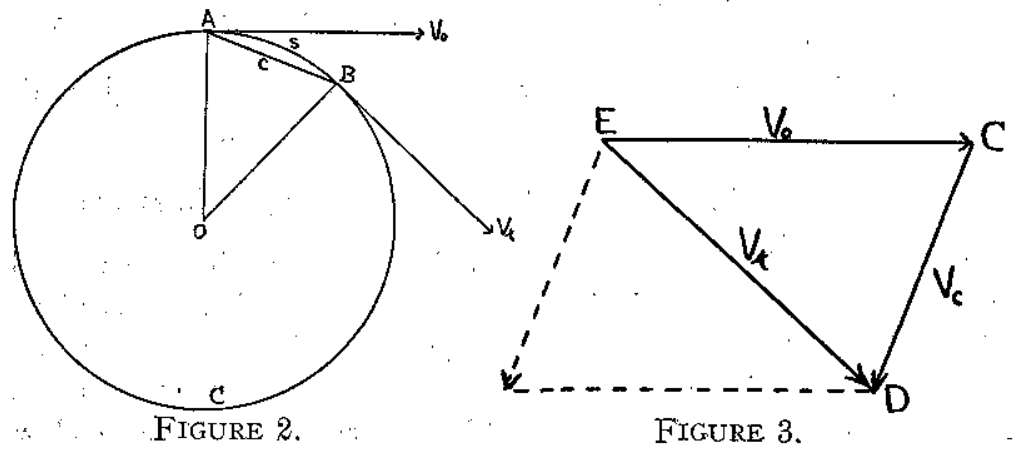

A body moving with uniform speed in the circular path ABCA, Figure 2, moves from $\mathrm{A}$ to $\mathrm{B}$ in $t$ seconds. The velocity of the body at point $A$ is represented, in both magnitude and direction, by the line $V_{0}$; also, the velocity at the point $B$ is fully represented by the line $V t$. The only change in velocity which takes place in the time $t$ is a change in the direction of the ve- 
locity, for the magnitude (speed in the path), is the same at $B$ as it is at A.

Fig. 3 shows that $V_{t}$ may be obtained by combining with $V_{\mathrm{O}}$ another velocity, $V_{c} ; i$. e., the velocity $V_{c}$, added to the velocity $V_{O}$, changes the direction of the resulting velocity without changing its magnitude. $\mathrm{Ve}$ is therefore the change in velocity in $t$ seconds.

Since $V_{O}$ in Figure 3 is perpendicular to $A O$ in Figure 2, and $V_{i}$ in Figure 3 is perpendicular to $O B$ in Figure 2. the triangles. $\mathrm{ABO}$ and $\mathrm{CDE}$ are similar and

$$
\frac{V_{c}}{C}=\frac{V_{0}}{r} \text { or } \frac{\text { Change in velocity }}{\text { Chord } \mathrm{C}}=\frac{\text { Velocity at } \mathrm{A}}{\text { Radius }} .
$$

It is clear that the velocity of the body is continually changing, and, since this change is a steady one, the rate at which the change takes place is the same for all points in the circular path. If the point $B$ is taken very near to the point $A$, the chord $\mathrm{C}$ and the arc $\mathrm{S}$ will be very nearly equal in length, and, with very small error, $\mathrm{S}$ may be written in place of C. Equation (3) then becomes $\frac{\mathrm{V} e}{\mathrm{~S}}=\frac{\mathrm{V}_{0}}{r} \quad$ (approximately).

But $S$ is the distance which the body travels in the very short interval of time, $t$, and the speed in the path is constant so that $\mathrm{S}=\mathrm{V}_{0} t$.

Substituting in (4),

$$
\frac{\mathrm{V} c}{\mathrm{~V}_{0} t}=\frac{\mathrm{V}_{0}}{r} \text { or } \frac{\mathrm{V} c}{t}=\frac{\mathrm{V}_{0}{ }^{2}}{r} \quad \text { (approximately). }
$$

But the change in velocity in time $t$, divided by $t$, gives the velocity change per unit time, $i . e$., the acceleration.

Therefore, the acceleration at $\mathrm{A}=\frac{\mathrm{V}_{0}^{2}}{r}$ (approximately)

But it is possible to make $t$ as small as we like, thus bringing $B$ nearer to $A$, and causing the chord $C$ to differ as little as we please from the arc $S$. Thus, as the change in velocity, $V c$, is expressed for a portion of the path less and less distant from the point $A$, expression (6) becomes more and more accurately true, and we are led to the conclusion that the acceleration at the point $\mathrm{A}$ is given exactly by the expression,

$$
a=\frac{\mathrm{V}_{0}{ }^{2}}{r}
$$

Where $a$ is the acceleration, $V^{\circ}$ is the velocity, in the circle, and $r$ is the radius of the circle. 
The student, in his study of electricity, meets with another case where he is asked to believe that the application of an assumption, not shown to be more than a mere approximation, leads to a perfectly exact result. The usual method of proof that the electrical potential at a distance $r$ from a charge $Q$ is expressed by the equation $\mathrm{V}=\frac{\mathrm{Q}}{d}$ involves an application of the method of limits.

The force acting on a unit charge at a point $P_{1}$ at a distance $d_{1}$ from $Q$ is $\frac{Q}{d_{1}^{2}}$; the force acting on a unit charge at $P_{2}$ at a distance $d_{2}$ from $Q$ is $\frac{Q}{d_{2}}$. The statement is made that if $P_{1}$ and $P_{2}$ are very near together, the average value of the force acting on a unit charge as it moves from one point to the other will be very nearly equal to the geometrical mean between the two quantities, namely, $\frac{Q}{d_{1} d_{2}}$. The validity of the proof rests upon the assumption that, in the limit, when $P_{2}$ is indefinitely close to $P_{1}$, the expression $\frac{Q}{d_{1} d_{2}}$ is actually the average force acting on the unit charge, yet the texts that use this form of proof inform the student that it is very nearly the average force. Thus, they not only do not justify the assumption made, but do not even clearly state it.

\title{
A NEW METHOD FOR THE MEASUREMENT OF THE WAVE LENGTH OF LIGHT WITH A COARSE GRATING.
}

\author{
By Will $\dot{\mathrm{C}}$. BaKer, \\ Queen's University, Kingston, Ont.
}

The following method has been used with satisfaction in this laboratory during the past few years.

Two rectangular pieces of brass $(10 \times 30 \mathrm{~cm}$.) are mounted against one another by means of brass straps that permit the sliding of one along the edge of the other. The amount of this displacement is controlled by a screw of millimetre pitch that is fitted with a head divided into twenty parts, as indicated in the figure. Two slits are cut in the brass as shown at $A, A$, and a section of one of these is given in the lower part of the sketch. A scale 